TRANSACTIONS OF THE

AMERICAN MATHEMATICAL SOCIETY

Volume 358, Number 3, Pages 1015-1031

S 0002-9947(05)03755-4

Article electronically published on March 31, 2005

\title{
HORROCKS THEORY AND THE BERNSTEIN-GEL'FAND-GEL'FAND CORRESPONDENCE
}

\author{
I. COANDĂ AND G. TRAUTMANN
}

\begin{abstract}
We construct an explicit equivalence between a category of complexes over the exterior algebra, which we call HT-complexes, and the stable category of vector bundles on the corresponding projective space, essentially translating into more fancy terms the results of Trautmann (1978) which, in turn, were influenced by ideas of Horrocks (1964), (1980). However, the result expressed by Theorem 5.1 and its corollary, which establishes a relation between the Tate resolutions over the exterior algebra (described in a paper by Eisenbud, Fløystad, and Schreyer) and HT-complexes, might be new, although, perhaps, not a surprise to experts.
\end{abstract}

\section{INTRODUCTION}

The Bernstein-Gel'fand-Gel'fand correspondence states that the derived category $\mathrm{D}^{b}\left(\mathrm{Coh} \mathbb{P}_{n}\right)$ of coherent sheaves on projective $n$-space is equivalent to the stable category of the category mod $-\Lambda$ of finitely generated graded modules over the exterior algebra $\Lambda=\Lambda^{\bullet} V$ of the corresponding vector space $V$ overlying $\mathbb{P}_{n}$; see 2]. New light was shed onto this correspondence by the paper [4] of EisenbudFløystad-Schreyer, who studied systematically the so-called Tate resolutions of sheaves or their graded modules. These Tate resolutions are doubly unbounded acyclic complexes of free graded $\Lambda$-modules; see Section 5 for a definition. The main result in [4] is that the terms of a Tate resolution of a sheaf are determined by its cohomology, as well as the linear parts of the differentials.

In [3, I. Coandă showed that a combination of a remark in 2] and the use of Tate resolutions over the exterior algebra leads to quick proofs of the main results of both 2 and 4 .

In 15, G. Trautmann had established a correspondence between complexes of extensions of Koszul operators and stable isomorphism classes of vector bundles on $\mathbb{P}_{n}$. This correspondence leads to applications on the structure of stable isomorphism classes of vector bundles analogous to the applications of the Beilinson monads. The complexes in [15] resemble filtrations studied in [10, and their construction was influenced by ideas of G. Horrocks.

In this paper, we show that the complexes in [15] correspond to bounded complexes $G^{\bullet}$ of free modules over the exterior algebra, of a structure similar to that of Tate resolutions, called HT-complexes; see Section 1.6. It is shown that the

Received by the editors December 11, 2003 and, in revised form, March 24, 2004.

2000 Mathematics Subject Classification. Primary 14F05, 15A75, 16E05.

The first author was partially supported by DFG and by CERES grant 152/2001 of the Romanian Ministry of Education and Research.

The research of the second author was supported by the DFG-Schwerpunktprogramm 1094.

(c)2005 American Mathematical Society

Reverts to public domain 28 years from publication 
BGG-functor L defines an equivalence between the homotopy category $\mathcal{H}$ of $\mathrm{HT}-$ complexes and the stable category of vector bundles over $\mathbb{P}_{n}$; see Section 2.2 In the course of proof, the results of [15] are re-proved. Furthermore, it is shown that an HT-complex $G^{\bullet}$ is determined up to isomorphism by the stable isomorphism class of the bundle it is defining.

The other main result of this paper is that an HT-complex $G^{\bullet}$ of a bundle is related to the Tate resolution $I^{\bullet}$ of the bundle by the formula $G^{\bullet}=F_{n-1} I^{\bullet} / F_{0} I^{\bullet}$; see Theorem 5.1. This reflects the fact that the cohomology $\mathrm{H}^{0}$ and $\mathrm{H}^{n}$ of bundles is neglected in the consideration of stable isomorphism classes.

We are grateful to Wolfram Decker for suggesting to us that the results from [15] could be related to the BGG correspondence via the results from [4 about Tate resolutions. We would like, however, to point out that, except for the proof of the comparison theorem, Theorem 5.1, our treatment of the subject is independent of the results from [2] and [4].

\section{1. $\Lambda$-MOdules AND ASSOCiated COMPleXes}

Notation. We shall use the notations and conventions from [3. It will also be convenient for us to refer to that paper for the proof of some standard facts.

Let $k$ be a field, let $V$ be an $(n+1)$-dimensional $k$-vector space, $n \geq 2$, and let $\mathbb{P}=\mathbb{P}_{n}(k)=\mathbb{P} V$ denote the projective space of 1-dimensional subspaces of $V$, such that $\mathrm{H}^{0} \mathcal{O}_{\mathbb{P} V}(1)=V^{*}$. We shall write $\mathcal{O}(d)=\mathcal{O}_{\mathbb{P}}(d)$ for the standard invertible sheaf on $\mathbb{P}$ of degree $d . \Lambda=\Lambda(V)=\bigoplus_{d \geq 0} \Lambda^{d} V$ denotes the exterior algebra of $V$ and $S=S\left(V^{*}\right)=\bigoplus_{d \geq 0} S^{d} V^{*}$ denotes the symmetric algebra of $V^{*}$.

The category of finitely generated graded right $\Lambda$-modules is denoted by $\bmod -\Lambda$.

The category $\mathrm{C}(\bmod -\Lambda)$ of complexes of modules in $\bmod -\Lambda$ will be denoted $\mathrm{C}(\Lambda)$, and the homotopy, resp. derived category of $\bmod -\Lambda$ by $\mathrm{K}(\Lambda)$, resp. $\mathrm{D}(\Lambda)$. Similarly, we use the notations $\mathrm{C}^{b}(\Lambda), \mathrm{K}^{b}(\Lambda)$ and $\mathrm{D}^{b}(\Lambda)$ for the categories of bounded complexes or $\mathrm{C}^{ \pm}(\Lambda), \mathrm{K}^{ \pm}(\Lambda), \mathrm{D}^{ \pm}(\Lambda)$ for the categories of half-bounded complexes.

Similarly we write $\mathrm{C}(\mathbb{P}), \mathrm{K}(\mathbb{P})$, and $\mathrm{D}(\mathbb{P})$ for the categories $\mathrm{C}(Q \operatorname{Coh}(\mathbb{P}))$, $\mathrm{K}(Q \operatorname{Coh}(\mathbb{P}))$, and $\mathrm{D}(Q \operatorname{Coh}(\mathbb{P}))$ based on the category of quasi-coherent sheaves over $\mathbb{P}$.

1.1. Free $\Lambda$-modules. The module $\Lambda$ has the natural positive grading with $\Lambda_{i}=$ $\Lambda^{i} V$, however, the dual module $\Lambda^{\vee}$ is defined by $\Lambda_{i}^{\vee}=\Lambda^{-i} V^{*}$. For an arbitrary $\Lambda_{-}^{-}$ module $N$ the dual $N^{\vee}$ is defined by $N_{i}^{\vee}=\left(N_{-i}\right)^{*}$. It is known (see, for example, [3, (4)) that an object $N$ of $\bmod -\Lambda$ is injective if and only if it is free, that is,

$$
N \cong \Lambda\left(a_{1}\right) \oplus \ldots \oplus \Lambda\left(a_{m}\right)
$$

or

$$
N \cong \Lambda^{\vee}\left(a_{1}\right) \oplus \ldots \oplus \Lambda^{\vee}\left(a_{m}\right)
$$

for some integers. Note that the isomorphisms $\Lambda^{p} V \rightarrow \operatorname{Hom}_{k}\left(\Lambda^{n+1-p} V, \Lambda^{n+1} V\right)$, $\omega \mapsto(-1)^{p}(-\wedge \omega)$, define an isomorphism $\Lambda \cong \Lambda^{\vee}(-n-1)$ in $\bmod -\Lambda$.

We let $\Lambda_{+} \subset \Lambda$ denote the ideal generated by $V$. Then $\Lambda / \Lambda_{+}^{i+1}$ is the module $k \oplus V \oplus \ldots \oplus \Lambda^{i} V$. It is easy to show (see, for example, [3], (4)(i)) that

1.2. Lemma. For any $N \in \mathrm{Ob}(\bmod -\Lambda)$ and any integer $a$, the natural map

$$
\operatorname{Hom}_{\bmod -\Lambda}\left(N, \Lambda^{\vee}(a)\right) \longrightarrow \operatorname{Hom}_{k}\left(N_{-a}, \Lambda^{\vee}(a)_{-a}\right)
$$

is bijective. 
1.3. The BGG-functor. To any $N \in \mathrm{Ob}(\bmod -\Lambda)$ one associates a bounded complex $\mathrm{L}(N) \in \mathrm{ObC}^{b}(\mathrm{Coh} \mathbb{P})$ defined by $\mathrm{L}(N)^{p}=N_{p} \otimes \mathcal{O}(p)$ with differential $N_{p} \otimes \mathcal{O}(p) \rightarrow N_{p+1} \otimes \mathcal{O}(p+1)$ induced by $N_{p} \otimes V \rightarrow N_{p+1}$, thus defining the BGG-functor

$$
\mathrm{L}: \bmod -\Lambda \longrightarrow \mathrm{C}^{b}(\mathrm{Coh} \mathbb{P})
$$

between $\bmod -\Lambda$ and the category of bounded complexes of coherent sheaves on $\mathbb{P}$. The complex $\mathrm{L}\left(\Lambda^{\vee}\right)$ is the standard Koszul complex

$$
0 \rightarrow \Lambda^{n+1} V^{*} \otimes \mathcal{O}(-n-1) \rightarrow \cdots \rightarrow \Lambda^{2} V^{*} \otimes \mathcal{O}(-2) \rightarrow V^{*} \otimes \mathcal{O}(-1) \rightarrow \mathcal{O} \rightarrow 0,
$$

and the complex $\mathrm{L}\left(\left(\Lambda / \Lambda_{+}^{i+1}\right)^{\vee}\right)$ is the truncation

$$
0 \longrightarrow \Lambda^{i} V^{*} \otimes \mathcal{O}(-i) \longrightarrow \cdots \longrightarrow V^{*} \otimes \mathcal{O}(-1) \longrightarrow \mathcal{O} \longrightarrow 0
$$

of the Koszul complex $\mathrm{L}\left(\Lambda^{\vee}\right)$ at $-i$.

Given a complex $K^{\bullet} \in \mathrm{Ob} \mathrm{C}(\Lambda)$, one obtains a double complex $X^{\bullet \bullet}$ of coherent sheaves on $\mathbb{P}$ with $X^{p q}=\mathrm{L}\left(K^{p}\right)^{q}$ and from this the simple complex

$$
\mathrm{L}\left(K^{\bullet}\right)=\mathrm{s}\left(X^{\bullet \bullet}\right)
$$

of the double complex, which is a complex of quasi-coherent sheaves. If $K^{\bullet}$ is bounded, then $\mathrm{L}\left(K^{\bullet}\right)$ is a bounded complex of coherent sheaves. In this way, the functor $\mathrm{L}$ can be extended to functors $\mathrm{C}(\Lambda) \rightarrow \mathrm{C}(\mathbb{P}), \mathrm{K}(\Lambda) \rightarrow \mathrm{K}(\mathbb{P}), \mathrm{D}^{+}(\Lambda) \rightarrow \mathrm{D}(\mathbb{P})$, and the last one restricts to a functor $\mathrm{D}^{b}(\Lambda) \rightarrow \mathrm{D}^{b}(\mathrm{Coh} \mathbb{P})$.

For later use we need the following lemma. For a short standard proof see 3, (5).

1.4. Lemma. Let $N^{\bullet} \in \mathrm{ObC}^{-}(\Lambda)$ be a complex of free (= injective) objects, bounded to the right. Then the complex $\mathrm{L}\left(N^{\bullet}\right)$ is acyclic. If, in addition, $N^{\bullet} \in$ $\mathrm{ObC}^{b}(\Lambda)$ is bounded, then $\mathrm{L}\left(N^{\bullet}\right)$ is bounded and consists of finite direct sums of line bundles.

\subsection{Definitions for complexes.}

(i) Given $N \in \mathrm{Ob}(\bmod -\Lambda)$ and an integer $d$, we denote by $N_{\leq d}$, resp. $N_{>d}$, the $\Lambda$-submodule of $N$ generated by its homogeneous elements of degree $\leq d$, resp. $\geq d$. Then $\left(N_{>d}\right)_{i}=N_{i}$ for $i \geq d$ and $=0$ for $i<d$.

If $N \stackrel{f}{\rightarrow} L$ is a homomorphism in $\bmod -\Lambda$ such that $\operatorname{Im}(f) \subset L \cdot \Lambda_{+}$, then $f\left(N_{\leq d}\right) \subset L_{\leq d-1}$.

(ii) We let $\mathcal{I}$ denote the full subcategory of $\bmod -\Lambda$ consisting of injective (= free) objects. If $I^{\bullet} \in \mathrm{ObC}(\mathcal{I})$, we may write each module as a finite sum

$$
I^{p}=\bigoplus_{i} H_{p-i}^{i} \otimes \Lambda^{\vee}(p-i),
$$

where $H_{p-i}^{i}$ are finite-dimensional $k$-vector spaces, the coefficient spaces of the modules $\Lambda^{\vee}(p-i)$. We say that $I^{\bullet}$ is minimal if $\operatorname{Im}\left(d_{I}^{p}\right) \subset I^{p+1} \cdot \Lambda_{+}$ for any $p$. This is equivalent to

$$
d_{I}^{p}\left(H_{p-i}^{i} \otimes \Lambda^{\vee}(p-i)\right) \subset \bigoplus_{j \leq i} H_{p+1-j}^{j} \otimes \Lambda^{\vee}(p+1-j)
$$

for any $p, i \in \mathbb{Z}$. This shows that, if $I^{\bullet}$ is minimal, the filtration on $I^{p}$, given by

$$
F_{j} I^{p}:=\bigoplus_{i \leq j} H_{p-i}^{i} \otimes \Lambda^{\vee}(p-i)
$$


for any index $p$, defines a subcomplex $F_{j} I^{\bullet}=\left(F_{j} I^{p}\right)_{p \in \mathbb{Z}}$ of $I^{\bullet}$. Alternatively, $F_{j} I^{p}=I_{\leq j-p-n-1}^{p}$. This filtration has already been considered by G. Fløystad [5]. The associated graded complex $\operatorname{gr}_{F}\left(I^{\bullet}\right)=\underset{j}{\oplus} F_{j} I^{\bullet} / F_{j-1} I^{\bullet}$ is the "linear part" of $I^{\bullet}$ as defined in 4 .

(iii) For any $K^{\bullet} \in \mathrm{ObC}(\Lambda)$ we define another subcomplex $\sigma K^{\bullet}$ of $K^{\bullet}$ by $\sigma K^{p}=$ $K_{\geq-p}^{p}$. If $I^{\bullet} \in \mathrm{ObC}(\mathcal{I})$ has the terms written as in (ii) above, then

$$
\sigma I^{p}=\bigoplus_{i} H_{p-i}^{i} \otimes\left(\Lambda / \Lambda_{+}^{i+1}\right)^{\vee}(p-i) .
$$

It follows from the definition of the functor $\mathrm{L}$ that

$$
\mathrm{L}\left(\sigma K^{\bullet}\right)=\sigma^{\geq 0} \mathrm{~L}\left(K^{\bullet}\right),
$$

where $\sigma^{\geq 0}$ denotes the "stupid" truncation of a complex, killing the terms of degree $<0$.

1.6. HT-complexes. A minimal bounded complex $G^{\bullet} \in \mathrm{Ob} \mathrm{C}^{b}(\mathcal{I})$ will be called an HT-complex if $F_{n-1} G^{\bullet}=G^{\bullet}$ and $F_{0} G^{\bullet}=0$. The modules of such a complex can be written as

$$
G^{p}=\bigoplus_{0<i<n} H_{p-i}^{i} \otimes \Lambda^{\vee}(p-i) .
$$

1.7. Lemma. For any $H T$-complex $G^{\bullet}$ the complex $\mathrm{L}\left(G^{\bullet}\right)$ is a bounded acyclic complex of finite direct sums of line bundles, and $\mathrm{L}\left(G^{\bullet}\right)^{p}=0$ for $p<-n$ or $p \geq n$.

The first statement follows from [1.4 the second from the special type of the modules $G^{p}$.

1.8. Remark. The sheaves of the complex $\mathrm{L}\left(G^{\bullet}\right)$ can be written as

$$
\mathrm{L}\left(G^{\bullet}\right)^{s}=\bigoplus_{0<i<n} \bigoplus_{d} H_{d}^{i} \otimes \Lambda^{i-s} V^{*} \otimes \mathcal{O}(-i-d+s)
$$

and so are precisely the terms of the acyclic complexes considered in [15. On the other hand, in [10] G. Horrocks employed filtrations of bundles $E \oplus L, L$ a direct sum of line bundles, which led to similar complexes. We let

$$
\mathcal{Z}^{0} \mathrm{~L}\left(G^{\bullet}\right)
$$

denote the kernel of the differential $\mathrm{L}\left(G^{\bullet}\right)^{0} \rightarrow \mathrm{L}\left(G^{\bullet}\right)^{1}$. One can verify that $\mathcal{Z}^{0} \mathrm{~L}\left(G^{\bullet}\right)$ is a successive extension of the sheaves $H_{d}^{i} \otimes \Omega^{i}(-d)$ and that $\mathrm{H}^{i}\left(\mathcal{Z}^{0} \mathrm{~L}\left(G^{\bullet}\right)(d)\right) \cong H_{d}^{i}$ for $0<i<n$ and any $d$. For the latter see Corollary 4.4 .

\section{The stable Category of Vector Bundles}

We denote by $\mathcal{H}$ the full subcategory of the homotopy category $\mathrm{K}(\Lambda)$ consisting of HT-complexes.

Let, furthermore, $\mathcal{V}$ denote the full subcategory of $\operatorname{Coh} \mathbb{P}(V)$ consisting of locally free sheaves (vector bundles) and $\mathcal{P}$ the full subcategory of $\mathcal{V}$ consisting of finite direct sums of line bundles. The stable category is the category $\mathcal{V} / \mathcal{P}$ which has the same objects as $\mathcal{V}$ but the sets Hom in $\mathcal{V} / \mathcal{P}$ are defined as

$$
\underline{\operatorname{Hom}}_{\mathcal{O}}\left(E, E^{\prime}\right):=\operatorname{Hom}_{\mathcal{O}}\left(E, E^{\prime}\right) / S\left(E, E^{\prime}\right) \text {, }
$$


where $S\left(E, E^{\prime}\right)$ is the subspace of homomorphisms which factorize through an object of $\mathcal{P}$. With this definition, a morphism $E \stackrel{f}{\rightarrow} E^{\prime}$ is an isomorphism in $\mathcal{V} / \mathcal{P}$ if and only if there are objects $P$ and $P^{\prime}$ in $\mathcal{P}$ such that $f$ factorises as

$$
E \hookrightarrow E \oplus P \stackrel{\approx}{\longrightarrow} E^{\prime} \oplus P^{\prime} \rightarrow E^{\prime}
$$

with an isomorphism in the middle. We need the following result of Horrocks:

2.1. Lemma. Let $E \stackrel{f}{\rightarrow} E^{\prime}$ be a homomorphism of vector bundles on $\mathbb{P}(V)$. If the induced homomorphisms $\mathrm{H}^{i} f(d)$ between $\mathrm{H}^{i} E(d)$ and $\mathrm{H}^{i} E^{\prime}(d)$ are isomorphisms for all $0<i<n$ and all $d$, then there exist direct sums $P$ and $P^{\prime}$ of line bundles such that $f$ decomposes as

$$
E \hookrightarrow E \oplus P \stackrel{\approx}{\longrightarrow} E^{\prime} \oplus P^{\prime} \rightarrow E^{\prime}
$$

This lemma is Theorem 7.5 in 9 . There, a short direct proof is given, independent of the other results in [9]. For the conclusion of Horrock's proof, it is convenient to consider the mapping cone of a certain quasi-isomorphism, arising naturally from his arguments.

2.2. Main result. The main result of this paper says that the functor

$$
G^{\bullet} \longmapsto \mathcal{Z}^{0} \mathrm{~L}\left(G^{\bullet}\right)
$$

induces an equivalence between the homotopy category $\mathcal{H}$ of HT-complexes and the stable category $\mathcal{V} / \mathcal{P}$.

This re-proves the main result of [15], saying that any vector bundle $E$ on $\mathbb{P}(V)$ is stably equivalent to a bundle $\mathcal{Z}^{0} \mathrm{~L}\left(G^{\bullet}\right)$.

The fact that the functor $\mathcal{H} \rightarrow \mathcal{V} / \mathcal{P}$ is fully faithful follows from Theorem 4.1 below, and the fact that it is essentially surjective from Theorem 5.1. Moreover, we shall prove that if $I^{\bullet} \stackrel{f^{\bullet}}{\longrightarrow} J^{\bullet}$ is a morphism of minimal complexes of injective objects of $\bmod -\Lambda$, then $f^{\bullet}$ is already an isomorphism if it is a homotopy equivalence; see Remark 4.2. This will imply that the (usual) isomorphism classes of HT-complexes are in bijection with the stable isomorphism classes of vector bundles on $\mathbb{P}(V)$.

\section{Auxiliary equivalences}

3.1. Lemma. Let $I^{\bullet} \in \mathrm{ObC}^{b}(\mathcal{I})$ be a bounded injective complex in $\bmod -\Lambda$ and suppose that $F_{0} I^{\bullet}=0$. Then, for any bounded complex $K^{\bullet} \in \mathrm{ObC}^{b}(\Lambda)$, the maps

$$
\operatorname{Hom}_{\mathrm{C}(\Lambda)}\left(K^{\bullet}, I^{\bullet}\right) \stackrel{\approx}{\longrightarrow} \operatorname{Hom}_{\mathrm{C}(\Lambda)}\left(\sigma K^{\bullet}, I^{\bullet}\right)
$$

and

$$
\operatorname{Hom}_{\mathrm{K}(\Lambda)}\left(K^{\bullet}, I^{\bullet}\right) \stackrel{\approx}{\underset{ }{\longrightarrow}} \operatorname{Hom}_{\mathrm{K}(\Lambda)}\left(\sigma K^{\bullet}, I^{\bullet}\right)
$$

are isomorphisms.

Proof. Let $\operatorname{Hom}^{\bullet}\left(K^{\bullet}, I^{\bullet}\right)$ denote the complex defined by

$$
\operatorname{Hom}^{q}\left(K^{\bullet}, I^{\bullet}\right)=\prod_{p} \operatorname{Hom}_{\bmod -\Lambda}\left(K^{p}, I^{p+q}\right)
$$

with differentials $d^{q}$ sending a tuple $\left(a^{p}\right)_{p}$ to $\left(a^{p+1} \circ d_{K}^{p}+(-1)^{q+1} d_{I}^{p+q} \circ a^{p}\right)_{p}$. Then $\operatorname{Hom}_{\mathrm{C}(\Lambda)}\left(K^{\bullet}, I^{\bullet}\right)=\operatorname{Ker} d^{0}$, and the morphisms $K^{\bullet} \rightarrow I^{\bullet}$ which are homotopically 
equivalent to zero are those of $\operatorname{Im} d^{-1}$. The statements of the lemma then follow if the maps

$$
\operatorname{Hom}^{i}\left(K^{\bullet}, I^{\bullet}\right) \stackrel{\approx}{\longrightarrow} \operatorname{Hom}^{i}\left(\sigma K^{\bullet}, I^{\bullet}\right)
$$

are isomorphisms for $i=-1,0,1$. But this follows easily from Lemma 1.2 .

3.2. Lemma. Let $I^{\bullet} \in \mathrm{ObC}^{b}(\mathcal{I})$ and $K^{\bullet} \in \mathrm{ObC}^{b}(\Lambda)$. Then the map

$$
\operatorname{Hom}_{\mathrm{K}(\Lambda)}\left(K^{\bullet}, I^{\bullet}\right) \stackrel{\approx}{\longrightarrow} \operatorname{Hom}_{\mathrm{K}(\mathbb{P})}\left(\mathrm{L}\left(K^{\bullet}\right), \mathrm{L}\left(I^{\bullet}\right)\right)
$$

is an isomorphism.

Proof. The functor $\operatorname{Hom}_{\mathrm{K}(\Lambda)}\left(-, I^{\bullet}\right)$ maps quasi-isomorphisms in $\mathrm{K}^{+}(\Lambda)$ to isomorphisms because $I^{\bullet}$ consists of injective objects, and L maps short exact sequences to semi-split short exact sequences. $\left(0 \rightarrow \mathcal{A}^{\bullet} \rightarrow \mathcal{B}^{\bullet} \rightarrow \mathcal{C}^{\bullet} \rightarrow 0\right.$ is called semi-split if each sequence $0 \rightarrow \mathcal{A}^{p} \rightarrow \mathcal{B}^{p} \rightarrow \mathcal{C}^{p} \rightarrow 0$ is split exact.)

Using the same kind of argument as at the beginning of the proof of [3], (6)(b), the proof can be reduced to the case where $K^{\bullet}=\underline{k}$ and then to the case where $I^{\bullet}=\mathrm{T}^{-p} \Lambda^{\vee}(p-i)$. In this last case, both sides are 0 , except when $p=i=0$, and, if $p=i=0$, the morphism of the lemma is clearly an isomorphism.

The next general fact should be well known. We include a sketch of the proof for lack of a reference.

3.3. Lemma. Let $\mathcal{A}$ be an abelian category with sufficiently many injective objects and let $X^{\bullet} \in \mathrm{ObC}^{-}(\mathcal{A})$ and $Y^{\bullet} \in \mathrm{ObC}^{+}(\mathcal{A})$ be bounded complexes to the right, resp. left. If $\operatorname{Ext}^{p-q}\left(X^{p}, Y^{q}\right)=0$ for all $p>q$, then the canonical map

$$
\operatorname{Hom}_{\mathrm{K}(\mathcal{A})}\left(X^{\bullet}, Y^{\bullet}\right) \longrightarrow \operatorname{Hom}_{\mathrm{D}(\mathcal{A})}\left(X^{\bullet}, Y^{\bullet}\right)
$$

is surjective, and if $\operatorname{Ext}^{p-q-1}\left(X^{p}, Y^{q}\right)=0$ for all $p>q+1$, then it is injective.

Proof. There is a quasi-isomorphism $v^{\bullet}: Y^{\bullet} \rightarrow J^{\bullet}$ with $J^{\bullet}$ bounded to the left and consisting of injective objects. It is well known that then the natural homomorphism

$$
\operatorname{Hom}_{\mathrm{K}(\mathcal{A})}\left(X^{\bullet}, J^{\bullet}\right) \stackrel{\approx}{\underset{ }{\longrightarrow}} \operatorname{Hom}_{\mathrm{D}(\mathcal{A})}\left(X^{\bullet}, J^{\bullet}\right)
$$

is an isomorphism for any complex $X^{\bullet}$ (any quasi-isomorphism $t^{\bullet}: J^{\bullet} \rightarrow Z^{\bullet}$ has a left inverse in $\mathrm{K}(\mathcal{A})$ because, the mapping cone $\operatorname{Con}\left(t^{\bullet}\right)$ being exact, $\left.\operatorname{Hom}_{\mathrm{K}(\mathcal{A})}\left(\mathrm{T}^{-1} \operatorname{Con}\left(t^{\bullet}\right), J^{\bullet}\right)=0\right)$. Also, because

$$
\operatorname{Hom}_{\mathrm{D}(\mathcal{A})}\left(X^{\bullet}, Y^{\bullet}\right) \cong \operatorname{Hom}_{\mathrm{D}(\mathcal{A})}\left(X^{\bullet}, J^{\bullet}\right),
$$

the statement of the lemma is equivalent to the surjectivity, resp. injectivity, of

$$
\operatorname{Hom}_{\mathrm{K}(\mathcal{A})}\left(X^{\bullet}, Y^{\bullet}\right) \longrightarrow \operatorname{Hom}_{\mathrm{K}(\mathcal{A})}\left(X^{\bullet}, J^{\bullet}\right) .
$$

In order to verify this, we consider the mapping cone $C^{\bullet}=\operatorname{Con}\left(v^{\bullet}\right)$ of $v^{\bullet}$, which is exact. We obtain the exact sequence

$$
\begin{aligned}
\operatorname{Hom}_{\mathrm{K}(\mathcal{A})}\left(X^{\bullet}, \mathrm{T}^{-1} C^{\bullet}\right) & \rightarrow \operatorname{Hom}_{\mathrm{K}(\mathcal{A})}\left(X^{\bullet}, Y^{\bullet}\right) \rightarrow \operatorname{Hom}_{\mathrm{K}(\mathcal{A})}\left(X^{\bullet}, J^{\bullet}\right) \\
& \rightarrow \operatorname{Hom}_{\mathrm{K}(\mathcal{A})}\left(X^{\bullet}, C^{\bullet}\right)
\end{aligned}
$$

The surjectivity of $(*)$ follows from $\operatorname{Hom}_{\mathrm{K}(\mathcal{A})}\left(X^{\bullet}, C^{\bullet}\right)=0$, which we prove next. Because $C^{q}=Y^{q+1} \oplus J^{q}$, the assumption of the lemma for surjectivity implies that $\operatorname{Ext}^{p-q}\left(X^{p}, C^{q-1}\right)=0$ for $p>q$. Because $C^{\bullet}$ is exact, there are the exact sequences

$$
0 \longrightarrow Z^{q-1}\left(C^{\bullet}\right) \longrightarrow C^{q-1} \longrightarrow Z^{q}\left(C^{\bullet}\right) \longrightarrow 0 .
$$


Using these, it follows that $\operatorname{Ext}^{1}\left(X^{p}, Z^{p-1}\left(C^{\bullet}\right)\right)$ injects into $\operatorname{Ext}^{2}\left(X^{p}, Z^{p-2}\left(C^{\bullet}\right)\right)$ which injects into $\operatorname{Ext}^{3}\left(X^{p}, Z^{p-3}\left(C^{\bullet}\right)\right)$ and so on. But $Z^{q}\left(C^{\bullet}\right) \subseteq C^{q}$ and $C^{q}=0$ for $q<<0$, hence $\operatorname{Ext}^{1}\left(X^{p}, Z^{p-1}\left(C^{\bullet}\right)\right)=0$. This implies that any morphism $X^{p} \rightarrow Z^{p}\left(C^{\bullet}\right)$ can be lifted to a morphism $X^{p} \rightarrow C^{p-1}$. This can finally be used to verify that any morphism $X^{\bullet} \rightarrow C^{\bullet}$ is homotopic to 0 , using descending induction and the assumption that $X^{\bullet}$ is bounded above. This proves that $(*)$ is surjective. The condition for injectivity implies, in the same way, that $\operatorname{Hom}_{\mathrm{K}(\mathcal{A})}\left(X^{\bullet}, \mathrm{T}^{-1} C^{\bullet}\right)=$ 0 .

\section{UNIQUENESS}

We are now in position to prove that an HT-complex $G^{\bullet}$ is determined by the stable isomorphism class of its associated sheaf $\mathcal{Z}^{0} \mathrm{~L}\left(G^{\bullet}\right)$ up to homotopy equivalence. More precisely, there is the

4.1. Theorem. Let $G^{\bullet}$ and $G^{\prime \bullet}$ be $H T$-complexes and let $\mathcal{E}=\mathcal{Z}^{0} \mathrm{~L}\left(G^{\bullet}\right)$ and $\mathcal{E}^{\prime}=\mathcal{Z}^{0} \mathrm{~L}\left(G^{\prime \bullet}\right)$ be the associated locally free sheaves. Then the map $f^{\bullet} \mapsto \mathcal{Z}^{0} \mathrm{~L}\left(f^{\bullet}\right)$ from

induces an isomorphism

$$
\operatorname{Hom}_{\mathrm{C}(\Lambda)}\left(G^{\bullet}, G^{\prime \bullet}\right) \longrightarrow \operatorname{Hom}_{\mathcal{O}}\left(\mathcal{E}, \mathcal{E}^{\prime}\right)
$$

$$
\operatorname{Hom}_{\mathrm{K}(\Lambda)}\left(G^{\bullet}, G^{\prime \bullet}\right) \stackrel{\approx}{\longrightarrow} \underline{\operatorname{Hom}}_{\mathcal{O}}\left(\mathcal{E}, \mathcal{E}^{\prime}\right)
$$

Proof. By Lemma 3.1 we have the isomorphisms

$$
\begin{aligned}
& \operatorname{Hom}_{\mathrm{C}(\Lambda)}\left(G^{\bullet}, G^{\prime \bullet}\right) \cong \operatorname{Hom}_{\mathrm{C}(\Lambda)}\left(\sigma G^{\bullet}, G^{\prime \bullet}\right), \\
& \operatorname{Hom}_{\mathrm{K}(\Lambda)}\left(G^{\bullet}, G^{\prime \bullet}\right) \cong \operatorname{Hom}_{\mathrm{K}(\Lambda)}\left(\sigma G^{\bullet}, G^{\prime \bullet}\right)
\end{aligned}
$$

and by Lemma 3.2 the isomorphism

$$
\operatorname{Hom}_{\mathrm{K}(\Lambda)}\left(\sigma G^{\bullet}, G^{\prime \bullet}\right) \cong \operatorname{Hom}_{\mathrm{K}(\mathbb{P})}\left(\mathrm{L}\left(\sigma G^{\bullet}\right), \mathrm{L}\left(G^{\prime \bullet}\right)\right) .
$$

Recalling $\mathrm{L}\left(\sigma G^{\bullet}\right)=\sigma^{\geq 0} \mathrm{~L}\left(G^{\bullet}\right)$, this is a right resolution of $\mathcal{E}$ by direct sums of line bundles, of length $n-1$; similarly for $\mathrm{L}\left(\sigma G^{\prime \bullet}\right)$. We, thus, obtain resolutions by direct sums of line bundles

$$
\begin{aligned}
& \mathcal{E}^{*} \longleftarrow \mathrm{L}\left(\sigma G^{\bullet}\right)^{*}, \\
& \mathcal{E}^{\prime *} \longleftarrow \mathrm{L}\left(\sigma G^{\prime \bullet}\right)^{*},
\end{aligned}
$$

of length $n-1$ and, therefore, induced free resolutions over the symmetric algebra S

$$
\begin{gathered}
\Gamma_{*} \mathcal{E}^{*} \longleftarrow \Gamma_{*}\left(\mathrm{~L}\left(\sigma G^{\bullet}\right)^{*}\right), \\
\Gamma_{*} \mathcal{E}^{\prime *} \longleftarrow \Gamma_{*}\left(\mathrm{~L}\left(\sigma G^{\prime \bullet}\right)^{*}\right) .
\end{gathered}
$$

Given a homomorphism $\mathcal{E} \stackrel{f}{\rightarrow} \mathcal{E}^{\prime}$ one can construct a homomorphism

$$
\Gamma_{*}\left(\mathrm{~L}\left(\sigma G^{\prime \bullet}\right)^{*}\right) \longrightarrow \Gamma_{*}\left(\mathrm{~L}\left(\sigma G^{\bullet}\right)^{*}\right)
$$

over $\Gamma_{*}\left(f^{*}\right)$ and, thus, a homomorphism

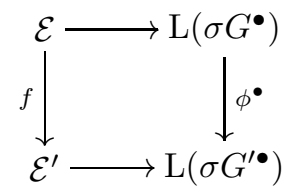


of the right resolutions over $f$. Now $\phi^{\bullet}$ can be extended by 0 into the left resolution $\sigma^{<0} \mathrm{~L}\left(G^{\prime \bullet}\right)$ of $\mathcal{E}^{\prime}$ to obtain a homomorphism $\mathrm{L}\left(\sigma G^{\bullet}\right) \rightarrow \mathrm{L}\left(G^{\prime \bullet}\right)$, hence a homomorphism $f^{\bullet}: \sigma G^{\bullet} \rightarrow G^{\prime \bullet}$. This proves that the map of the theorem is surjective.

If $\sigma G^{\bullet} \rightarrow G^{\prime \bullet}$ is homotopic to zero, then the induced homomorphism $\mathcal{E} \rightarrow \mathcal{E}^{\prime}$ factorizes through $\mathrm{L}\left(G^{\prime \bullet}\right)^{-1}$ and, thus, becomes zero in the stable category. Let, conversely, $\sigma G^{\bullet} \stackrel{f^{\bullet}}{\longrightarrow} G^{\prime \bullet}$ define a homomorphism $\mathcal{E} \stackrel{f}{\rightarrow} \mathcal{E}^{\prime}$, which factorizes through a direct sum $\mathcal{L}$ of line bundles. Because $T^{-1} \sigma^{<0} \mathrm{~L}\left(G^{\prime \bullet}\right)$ is a left resolution of $\mathcal{E}^{\prime}$ by direct sums of line bundles, of length $n-1$, we obtain a free resolution over the symmetric algebra $S$

$$
\Gamma_{*} T^{-1} \sigma^{<0} \mathrm{~L}\left(G^{\prime \bullet}\right) \longrightarrow \Gamma_{*} \mathcal{E}^{\prime}
$$

and then $\Gamma_{*} \mathcal{L} \rightarrow \Gamma_{*} \mathcal{E}^{\prime}$ factorizes through $\Gamma_{*} \mathrm{~L}\left(G^{\bullet}\right)^{-1}$. Using the dual resolutions again, one can construct from this a homotopy $\Gamma_{*}\left(\mathrm{~L}\left(f^{\bullet}\right)^{*}\right) \sim 0$, hence $\mathrm{L}\left(f^{\bullet}\right)^{*} \sim 0$, hence $\mathrm{L}\left(f^{\bullet}\right) \sim 0$. It follows that $f^{\bullet} \sim 0$. This proves that

$$
\operatorname{Hom}_{\mathrm{K}(\Lambda)}\left(G^{\bullet}, G^{\prime \bullet}\right) \rightarrow \underline{\operatorname{Hom}}_{\mathcal{O}}\left(\mathcal{E}, \mathcal{E}^{\prime}\right)
$$

is also injective.

4.2. Remark. Let $I^{\bullet}$ and $J^{\bullet}$ be minimal complexes in $\mathrm{C}(\mathcal{I})$ and let $I^{\bullet} \stackrel{f^{\bullet}}{\longrightarrow} J^{\bullet}$ be a morphism of complexes. If $f^{\bullet}$ is a homotopy equivalence (i.e., an isomorphism in $\mathrm{K}(\Lambda)$ ), then it is already an isomorphism in $\mathrm{C}(\Lambda)$.

Proof. Because all modules $I^{p}$ and $J^{p}$ are free, we may again write $I^{p}=\bigoplus_{i} H_{p-i}^{i} \otimes$ $\Lambda^{\vee}(p-i)$ and $J^{p}=\bigoplus_{i} K_{p-i}^{i} \otimes \Lambda^{\vee}(p-i) . f^{\bullet}$ induces isomorphisms

$$
\operatorname{Hom}_{\mathrm{K}(\Lambda)}\left(\underline{k}, \mathrm{~T}^{p} I^{\bullet}(i-p)\right) \stackrel{\approx}{\longrightarrow} \operatorname{Hom}_{\mathrm{K}(\Lambda)}\left(\underline{k}, \mathrm{~T}^{p} J^{\bullet}(i-p)\right)
$$

for all $p, i \in \mathbb{Z}$, where $\underline{k}=\Lambda / \Lambda_{+}$. Since $I^{\bullet}$ is minimal,

$$
\operatorname{Hom}_{\mathrm{K}(\Lambda)}\left(\underline{k}, \mathrm{~T}^{p} I^{\bullet}(i-p)\right) \cong H_{p-i}^{i} \otimes\left(\Lambda^{\vee}\right)_{0}
$$

and, analogously, for the other term. One deduces that the component of $f_{i-p}^{p}$ mapping $H_{p-i}^{i} \otimes \Lambda^{\vee}(p-i)_{i-p}$ to $K_{p-i}^{i} \otimes \Lambda^{\vee}(p-i)_{i-p}$ is an isomorphism, hence the component of $f^{p}$ mapping $H_{p-i}^{i} \otimes \Lambda^{\vee}(p-i)$ to $K_{p-i}^{i} \otimes \Lambda^{\vee}(p-i)$ is an isomorphism for all $p, i \in \mathbb{Z}$. It follows that each $f^{p}$ is an isomorphism (because the matrix defining it is triangular).

The following isomorphism will be needed later. Its corollary is the result 3.7 in [15].

4.3. Lemma. Let $I^{\bullet} \in \mathrm{ObC}^{b}(\mathcal{I})$ and let $\underline{k}=\Lambda / \Lambda_{+}$. Then the natural homomorphism

$$
\operatorname{Hom}_{\mathrm{K}(\Lambda)}\left(\underline{k},\left(\sigma I^{\bullet}\right)(m)\right) \stackrel{\approx}{\longrightarrow} \operatorname{Hom}_{\mathrm{D}(\mathbb{P})}\left(\mathrm{L}(\underline{k}), \mathrm{L}\left(\left(\sigma I^{\bullet}\right)(m)\right)\right)
$$

is an isomorphism for $0<m<n$.

Proof. $\left(\sigma I^{p}\right)(m)_{0}=I^{p}(m)_{0}$ for $p \geq-1$ and $m>0$ and so

$$
\operatorname{Hom}_{\mathrm{K}(\Lambda)}\left(\underline{k},\left(\sigma I^{\bullet}\right)(m)\right) \cong \operatorname{Hom}_{\mathrm{K}(\Lambda)}\left(\underline{k}, I^{\bullet}(m)\right) .
$$

As in 1.5. we have $\mathrm{L}\left(\left(\sigma I^{\bullet}\right)(m)\right)=\sigma^{\geq-m} \mathrm{~L}\left(I^{\bullet}(m)\right)$. Therefore, also,

$$
\operatorname{Hom}_{\mathrm{K}(\mathbb{P})}\left(\mathrm{L}(\underline{k}), \mathrm{L}\left(\left(\sigma I^{\bullet}\right)(m)\right)\right) \cong \operatorname{Hom}_{\mathrm{K}(\mathbb{P})}\left(\mathrm{L}(\underline{k}), \mathrm{L}\left(I^{\bullet}(m)\right)\right)
$$


for $m>0$. Now Lemma 3.2 applied to the free (= injective) complex $I^{\bullet}(m)$ implies that the natural homomorphism

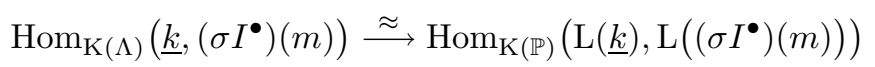

is an isomorphism. Because $\mathrm{L}(\underline{k})=\mathcal{O}$, the conditions of Lemma 3.3 are satisfied for $m<n$ so that the last $\operatorname{Hom}_{\mathrm{K}(\mathbb{P})}$ is isomorphic to $\operatorname{Hom}_{\mathrm{D}(\mathbb{P})}$.

4.4. Corollary. If $G^{\bullet}$ is an $H T$-complex, then $\mathrm{H}^{m}\left(\mathcal{Z}^{0} \mathrm{~L}\left(G^{\bullet}\right)(p-m)\right) \cong H_{p-m}^{m}$ for $0<m<n$ and all $p \in \mathbb{Z}$.

Proof. It follows from the definition of the functor $\mathrm{L}$ that $\mathrm{L}\left(\mathrm{T}^{p}\left(\sigma G^{\bullet}\right)(m-p)\right)=$ $\mathrm{T}^{m} \mathrm{~L}\left(\sigma G^{\bullet}\right)(p-m)$. Applying the previous lemma to $I^{\bullet}=\mathrm{T}^{p} G^{\bullet}(-p)$ we obtain (because $\sigma I^{\bullet}=\mathrm{T}^{p}\left(\sigma G^{\bullet}\right)(-p)$ ) an isomorphism

$$
\operatorname{Hom}_{\mathrm{K}(\Lambda)}\left(\underline{k}, \mathrm{~T}^{p}\left(\sigma G^{\bullet}\right)(m-p)\right) \cong \operatorname{Hom}_{\mathrm{D}(\mathbb{P})}\left(\mathrm{L}(\underline{k}), \mathrm{T}^{m} \mathrm{~L}\left(\sigma G^{\bullet}\right)(p-m)\right) .
$$

Now it is easy to verify that the left-hand side is isomorphic to $H_{p-m}^{m}$. Because $\mathrm{L}\left(\sigma G^{\bullet}\right)$ is quasi-isomorphic to the trivial complex $\mathcal{E}=\mathcal{Z}^{0} \mathrm{~L}\left(G^{\bullet}\right)$, the right-hand side is isomorphic to

$$
\operatorname{Hom}_{\mathrm{D}(\mathbb{P})}\left(\mathcal{O}, \mathrm{T}^{m} \mathcal{E}(p-m)\right)=\operatorname{Ext}^{m}(\mathcal{O}, \mathcal{E}(p-m)) \cong \mathrm{H}^{m} \mathcal{E}(p-m) .
$$

4.5. Remark. One can show, moreover, that the linear part $H_{p-m}^{m} \otimes \Lambda^{\vee}(p-m) \rightarrow$ $H_{p+1-m}^{m} \otimes \Lambda^{\vee}(p+1-m)$ of the differential $d_{G}^{m}$ is induced (up to sign) by the multiplication map $\mathrm{H}^{m} \mathcal{E}(p-m) \otimes V^{*} \rightarrow \mathrm{H}^{m} \mathcal{E}(p+1-m)$.

Indeed, let $I^{\bullet} \in \mathrm{ObC}^{b}(\mathcal{I})$ and $0<m<n$. We have seen, in the proof of Lemma 4.3, that one has a canonical isomorphism

$$
\operatorname{Hom}_{\mathrm{K}(\Lambda)}\left(\underline{k}, I^{\bullet}(m)\right) \cong \operatorname{Hom}_{\mathrm{D}(\mathbb{P})}\left(\mathrm{L}(\underline{k}), \mathrm{L}\left(\left(\sigma I^{\bullet}\right)(m)\right)\right)
$$

and, in the same way, one proves that there is a canonical isomorphism

$$
\operatorname{Hom}_{\mathrm{K}(\Lambda)}\left(\mathrm{T}^{-1} \underline{k}(1), I^{\bullet}(m)\right) \cong \operatorname{Hom}_{\mathrm{D}(\mathbb{P})}\left(\mathrm{L}\left(\mathrm{T}^{-1} \underline{k}(1)\right), \mathrm{L}\left(\left(\sigma I^{\bullet}\right)(m)\right)\right) .
$$

Now, one may use the arguments from the proof of $[3],(10)(b)$.

For the proof of Theorem 5.1 below we also need the following lemma which is [2, Remark 3 after Theorem 2, in the form stated and proved in [3], (6)(b).

4.6. Lemma. Let $I^{\bullet} \in \mathrm{ObC}(\mathcal{I})$ be an acyclic complex. Then the canonical morphism

$$
\operatorname{Hom}_{\mathrm{K}(\Lambda)}\left(\underline{k}, I^{\bullet}\right) \stackrel{\approx}{\longrightarrow} \operatorname{Hom}_{\mathrm{D}(\mathbb{P})}\left(\mathrm{L}(\underline{k}), \mathrm{L}\left(I^{\bullet}\right)\right)
$$

is an isomorphism.

\section{Comparison with Tate Resolutions}

It was shown in 15 that any stable isomorphism class of a vector bundle $\mathcal{E}$ on $\mathbb{P} V$ is the class of $\mathcal{Z}^{0} \mathrm{~L}\left(G^{\bullet}\right)$ of an HT-complex $G^{\bullet}$ with $H_{p-i}^{i}=\mathrm{H}^{i} \mathcal{E}(p-i)$ for $0<i<n$, and that this complex is unique up to isomorphisms. On the other hand, the main result of [2] was that there is a module $N \in \mathrm{Ob}(\bmod -\Lambda)$, annihilated by $\operatorname{soc}(\Lambda)=\Lambda_{n+1}$, such that $\mathrm{L}(N)$ is a monad for $\mathcal{E}$, that is, $\mathcal{H}^{0} \mathrm{~L}(N) \cong \mathcal{E}$ and $\mathcal{H}^{i} \mathrm{~L}(N)=0$ for $i \neq 0$. Also in this situation $N$ is unique up to isomorphism. In [7], and recently in [4, so-called Tate resolutions of $\Lambda$-modules associated to bundles or coherent sheaves on $\mathbb{P} V$ have been used in order to improve the understanding of the result of [2]. A Tate resolution in our setting is an acyclic minimal complex $I^{\bullet}$ 
in $\mathrm{C}(\mathcal{I})$ such that $N \cong Z^{0}\left(I^{\bullet}\right)=\operatorname{Ker}\left(I^{0} \rightarrow I^{1}\right)$. We say that $I^{\bullet}$ is a Tate resolution of $\mathcal{E}$. This agrees with the definition in [4, Section 4, as one may verify by using [3], (10). The main result in [4] is that

$$
I^{p} \cong \bigoplus_{0 \leq i \leq n} \mathrm{H}^{i} \mathcal{E}(p-i) \otimes \Lambda^{\vee}(p-i)
$$

and that the linear part

$$
\mathrm{H}^{i} \mathcal{E}(p-i) \otimes \Lambda^{\vee}(p-i) \longrightarrow \mathrm{H}^{i} \mathcal{E}(p+1-i) \otimes \Lambda^{\vee}(p+1-i)
$$

of the differential $I^{p} \rightarrow I^{p+1}$ is induced by the multiplication map (also valid for hyper-cohomology of a complex $\mathcal{F}^{\bullet}$ of coherent sheaves). This was stated in 2], Remark 3 after Theorem 2, and completely proved in [4]; see also [3] for an exposition of these results in a spirit closer to that of 2 .

The relation between HT-complexes $G^{\bullet}$ and the Tate resolutions for a bundle $\mathcal{E}$ is described in the following theorem and its corollary.

5.1. Theorem. Let $\mathcal{E}$ be a vector bundle on $\mathbb{P}(V)$ and let $I^{\bullet}$ be a Tate resolution of $\mathcal{E}$. Then $\mathcal{E}$ is stably isomorphic to $\mathcal{Z}^{0} \mathrm{~L}\left(F_{n-1} I^{\bullet} / F_{0} I^{\bullet}\right)$.

Proof. (1) We may assume that $I^{p}=\bigoplus_{i} H_{p-i}^{i} \otimes \Lambda^{\vee}(p-i)$ with $H_{d}^{i}=\mathrm{H}^{i} \mathcal{E}(d)$. It follows that

$$
G^{\bullet}:=F_{n-1} I^{\bullet} / F_{0} I^{\bullet}
$$

is an HT-complex. $G^{\bullet}$ is a subcomplex of $I^{\bullet} / F_{0} I^{\bullet}$ but not necessarily of $I^{\bullet}$. However, $\sigma G^{\bullet}$ is a subcomplex of $I^{\bullet}$ because

$$
\sigma G^{p}=\bigoplus_{0<i<n} H_{p-i}^{i} \otimes\left(\Lambda / \Lambda_{+}^{i+1}\right)^{\vee}(p-i)
$$

and any morphism $\left(\Lambda / \Lambda_{+}^{i+1}\right)^{\vee}(p-i) \rightarrow \Lambda^{\vee}(p+1)$ is 0 by 1.2 .

(2) The complex $\mathrm{L}\left(I^{\bullet}\right)$ consists of quasi-coherent sheaves which are at most countable direct sums of line bundles, $\mathcal{H}^{0} \mathrm{~L}\left(I^{\bullet}\right) \cong \mathcal{E}$ and $\mathcal{H}^{i} \mathrm{~L}\left(I^{\bullet}\right)=0$ for $i \neq 0$ by [3], (6)(a).

(3) Now let $\mathcal{F}=\mathcal{Z}^{0} \mathrm{~L}\left(G^{\bullet}\right)=\mathcal{Z}^{0} \mathrm{~L}\left(\sigma G^{\bullet}\right)$. Because $\mathrm{L}\left(\sigma G^{\bullet}\right)$ is a right resolution of $\mathcal{F}$, the inclusion $\sigma G^{\bullet} \hookrightarrow I^{\bullet}$ induces a homomorphism $\mathcal{F} \stackrel{f}{\rightarrow} \mathcal{E}$ via $\mathrm{L}\left(\sigma G^{\bullet}\right) \rightarrow \mathrm{L}\left(I^{\bullet}\right)$. We are going to show that $f$ is the stable isomorphism of the theorem. For that, we consider the diagram

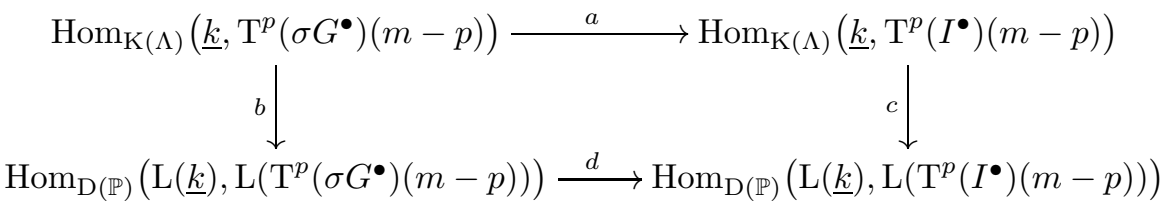

for any $p \in \mathbb{Z}$ and $0<m<n$. The map $a$ is an isomorphism because a map $\underline{k} \rightarrow \mathrm{T}^{p}\left(\sigma G^{\bullet}\right)(m-p)$ can have its target only in $H_{p-m}^{m} \otimes \underline{k}$ and, similarly, for $I^{\bullet}$, and because $I^{\bullet}$ is minimal. The map $b$ is an isomorphism by Lemma 4.3, and $c$ is an isomorphism by Lemma 4.6. It follows that $d$ is an isomorphism. But $d$ can be identified with $\mathrm{H}^{m} f(p-m): \mathrm{H}^{m} \mathcal{F}(p-m) \rightarrow \mathrm{H}^{m} \mathcal{E}(p-m)$. Now Lemma 2.1 implies that $f$ is an isomorphism of the stable classes of $\mathcal{F}$ and $\mathcal{E}$. 
5.2. Corollary. Let $G^{\bullet}$ be any HT-complex, let $\mathcal{E}=\mathcal{Z}^{0} \mathrm{~L}\left(G^{\bullet}\right)$ be its associated bundle and let $I^{\bullet}$ be the Tate resolution of $\mathcal{E}$. Then

$$
G^{\bullet} \cong F_{n-1} I^{\bullet} / F_{0} I^{\bullet}
$$

in the category $\mathrm{C}(\Lambda)$ of complexes.

Proof. Let $\mathcal{F}=\mathcal{Z}^{0} \mathrm{~L}\left(F_{n-1} I^{\bullet} / F_{0} I^{\bullet}\right)$ as in the previous proof. By the theorem, $\mathcal{E}$ and $\mathcal{F}$ are stably equivalent. Then, by Theorem 4.1 , there is a homotopy equivalence $G^{\bullet} \stackrel{f^{\bullet}}{\longrightarrow} F_{n-1} I^{\bullet} / F_{0} I^{\bullet}$. By Remark 4.2, $f^{\bullet}$ must be an isomorphism of complexes.

5.3. Remark. Let $I^{\bullet} \in \mathrm{ObC}^{b}(\mathcal{I})$ be a minimal bounded complex of injective objects of $\bmod -\Lambda$, and let $\mathcal{E}=\mathcal{Z}^{0} \mathrm{~L}\left(I^{\bullet}\right)$. Then $\mathcal{E}$ is stably isomorphic to

$$
\mathcal{Z}^{0} \mathrm{~L}\left(F_{n-1} I^{\bullet} / F_{0} I^{\bullet}\right) \text {. }
$$

Indeed, one can repeat the arguments from the proof of Theorem 5.1, using Lemma 4.3 instead of Lemma 4.6

\section{HT-RESOlutions}

Each stable isomorphism class of a vector bundle contains a unique element, which has no direct summand of rank 1 . The next result, which was proved in [15, Section 8 , shows how one can compute the invariants of this bundle in terms of the corresponding HT-complex.

6.1. Proposition. Let $G^{\bullet}$ be an $H T$-complex, with $G^{p}=\underset{0<i<n}{\bigoplus} H_{p-i}^{i} \otimes \Lambda^{\vee}(p-i)$. Then

(a) $\operatorname{rank} \mathcal{Z}^{0} \mathrm{~L}\left(G^{\bullet}\right)=\sum_{0<i<n}\left(\begin{array}{c}n \\ i\end{array}\right) \sum_{p} \operatorname{dim}\left(H_{p-i}^{i}\right)$.

(b) If $r(p)$ denotes the rank of $G_{-p}^{p-1} \rightarrow G_{-p}^{p}$, then $\mathcal{Z}^{0} \mathrm{~L}\left(G^{\bullet}\right) \cong \mathcal{E} \oplus \bigoplus_{p} \mathcal{O}(-p)^{r(p)}$, where $\mathcal{E}$ contains no direct summand of rank 1.

Proof. (a) rank $\mathcal{Z}^{0} \mathrm{~L}\left(G^{\bullet}\right)$ is an additive function with respect to $G^{\bullet}$, hence we may assume that $G^{\bullet}=\mathrm{T}^{-p} \Lambda^{\vee}(p-i)$. In this case, $\mathcal{Z}^{0} \mathrm{~L}\left(G^{\bullet}\right)=\Omega_{\mathbb{P}}^{i}(i-p)$.

(b) Note that the map $d_{G,-p}^{p-1}$ between the vector spaces $G_{-p}^{p-1}$ and $G_{-p}^{p}$ decomposes into

$$
\bigoplus_{0<i<n} H_{p-1-i}^{i} \otimes \Lambda^{i+1} V^{*} \longrightarrow \bigoplus_{0<i<n} H_{p-i}^{i} \otimes \Lambda^{i} V^{*}
$$

We have

$$
\mathrm{L}\left(G^{\bullet}\right)^{-1}=\bigoplus_{p} G_{-p}^{p-1} \otimes \mathcal{O}(-p) \quad \text { and } \quad \mathrm{L}\left(G^{\bullet}\right)^{0}=\bigoplus_{p} G_{-p}^{p} \otimes \mathcal{O}(-p) .
$$

We are going to cancel the constant part of the differential $d_{\mathrm{L}(G)}^{-1}: \mathrm{L}\left(G^{\bullet}\right)^{-1} \rightarrow$ $\mathrm{L}\left(G^{\bullet}\right)^{0}$. Let $Z^{p-1}$ (resp. $B^{p}$ ) be the kernel (resp. image) of $d_{G}^{p-1}: G^{p-1} \rightarrow G^{p}$. In degree $-p$, we choose decompositions

$$
Y_{-p}^{p-1} \oplus Z_{-p}^{p-1}=G_{-p}^{p-1} \quad \text { and } \quad B_{-p}^{p} \oplus C_{-p}^{p}=G_{-p}^{p}
$$


such that $Y_{-p}^{p-1} \cong B_{-p}^{p}$ under $d_{G}^{p-1}$. Then the differential $\mathrm{L}\left(G^{\bullet}\right)^{-1} \rightarrow \mathrm{L}\left(G^{\bullet}\right)^{0}$ decomposes into

$$
\left(\begin{array}{l}
\alpha \beta \\
\gamma \delta
\end{array}\right): \mathcal{Y} \oplus \mathcal{G}^{-1} \longrightarrow \mathcal{B} \oplus \mathcal{G}^{0}
$$

where the sheaves in this decomposition are defined according to the above decomposition, i.e., $\mathcal{G}^{-1}=\bigoplus_{p} Z_{-p}^{p-1} \otimes \mathcal{O}(-p), \mathcal{G}^{0}=\bigoplus_{p} C_{-p}^{p} \otimes \mathcal{O}(-p)$ and, similarly, $\mathcal{Y}$ and $\mathcal{B}$. Since $\alpha$ is an isomorphism, we may consider the automorphisms

$$
\Phi=\left(\begin{array}{cc}
\text { id } & 0 \\
\gamma^{\prime} & \text { id }
\end{array}\right): \mathrm{L}\left(G^{\bullet}\right)^{0} \stackrel{\approx}{\longrightarrow} \mathrm{L}\left(G^{\bullet}\right)^{0} \quad \text { and } \quad \Psi=\left(\begin{array}{cc}
\text { id } \beta^{\prime} \\
0 & \text { id }
\end{array}\right): \mathrm{L}\left(G^{\bullet}\right)^{-1} \stackrel{\approx}{\longrightarrow} \mathrm{L}\left(G^{\bullet}\right)^{-1},
$$

where $\gamma^{\prime}=-\gamma \alpha^{-1}$ and $\beta^{\prime}=-\alpha^{-1} \beta$. Then we have

$$
\Phi \circ d_{\mathrm{L}(G)}^{-1} \circ \Psi=\left(\begin{array}{cc}
\alpha & 0 \\
0 & \delta^{\prime}
\end{array}\right),
$$

where $\delta^{\prime}=\delta-\gamma \alpha^{-1} \beta: \mathcal{G}^{-1} \rightarrow \mathcal{G}^{0}$. Since $d_{\mathrm{L}(G)}^{-1}$ maps $Z_{-p}^{p-1} \otimes \mathcal{O}(-p)$ to $\bigoplus_{q<p} G_{-q}^{q} \otimes$ $\mathcal{O}(-q), \delta^{\prime}$ maps each $Z_{-p}^{p-1} \otimes \mathcal{O}(-p)$ into $\bigoplus_{q<p} C_{-q}^{q} \otimes \mathcal{O}(-q)$. This means that $\delta^{\prime}$ has no constant component. We have an induced acyclic complex

$$
\cdots \longrightarrow \mathrm{L}\left(G^{\bullet}\right)^{-2} \longrightarrow \mathcal{G}^{-1} \stackrel{\delta^{\prime}}{\longrightarrow} \mathcal{G}^{0} \longrightarrow \mathrm{L}\left(G^{\bullet}\right)^{1} \longrightarrow \cdots,
$$

where $\mathrm{L}\left(G^{\bullet}\right)^{-2} \rightarrow \mathcal{G}^{-1}$ and $\mathcal{G}^{0} \rightarrow \mathrm{L}\left(G^{\bullet}\right)^{1}$ are just the components of $d_{\mathrm{L}(G)}^{-2}$ and $d_{\mathrm{L}(G)}^{0}$, respectively. Let $\mathcal{E}$ denote the image of $\delta^{\prime}$. Then

$$
\mathcal{E} \oplus \bigoplus_{p} B_{-p}^{p} \otimes \mathcal{O}(-p)=\mathcal{E} \oplus \mathcal{B}=\operatorname{Im}\left(\Phi \circ d_{\mathrm{L}(G)}^{-1} \circ \Psi\right) \cong \operatorname{Im} d_{\mathrm{L}(G)}^{-1}=\mathcal{Z}^{0} \mathrm{~L}\left(G^{\bullet}\right) .
$$

It remains to show that $\mathcal{E}$ has no direct summand of rank 1 . Suppose there is such a summand. Then we have a decomposition $\mathcal{O} \stackrel{\rho}{\rightarrow} \mathcal{E}(a) \stackrel{\pi}{\rightarrow} \mathcal{O}$ with $\pi \circ \rho=$ id for some integer $a$. Because the left resolution of $\mathcal{E}$ has length $n-1, \Gamma \mathcal{G}^{-1}(a) \rightarrow \Gamma \mathcal{E}(a)$ is surjective and $\rho$ factorizes through $\mathcal{G}^{-1}(a)$. By the same argument applied to $\mathcal{E}^{*}$, $\mathcal{E}(a) \rightarrow \mathcal{O}$ also extends to $\mathcal{G}^{0}(a) \rightarrow \mathcal{O}$. But this would imply that $\mathcal{G}^{-1} \rightarrow \mathcal{G}^{0}$ has a non-zero component $\mathcal{O}(-a) \rightarrow \mathcal{O}(-a)$, contradicting the property of $\delta^{\prime}$ stated above.

6.2. Remark. By pursuing the process of cancelation of constant parts of the differentials, we eventually obtain from the complex $\mathrm{L}\left(G^{\bullet}\right)$ an acyclic complex

$$
0 \longrightarrow \mathcal{L}^{-n} \longrightarrow \cdots \longrightarrow \mathcal{L}^{-1} \longrightarrow \mathcal{L}^{0} \longrightarrow \cdots \longrightarrow \mathcal{L}^{n-1} \longrightarrow 0
$$

with terms

$$
\mathcal{L}^{m} \cong \bigoplus_{p} H^{p}\left(G^{\bullet}\right)_{m-p} \otimes \mathcal{O}(m-p),
$$

whose differentials have no constant part, and such that $\mathcal{E} \cong \mathcal{Z}^{0} \mathcal{L}^{\bullet}$. One has $H^{i}\left(\Gamma_{*} \mathcal{L}^{\bullet}\right) \cong \mathrm{H}_{*}^{i} \mathcal{E}$ as graded $S$-modules for $0<i<n$, and $H^{i}\left(\Gamma_{*} \mathcal{L}^{\bullet}\right)=0$ for $i \leq 0$.

Moreover, the "linear part" of $\mathcal{L}^{\bullet}$ is isomorphic to $\mathrm{L}\left(H^{\bullet}\left(G^{\bullet}\right)\right)$, where $H^{\bullet}\left(G^{\bullet}\right)$ is the complex whose $p$ th term is $H^{p}\left(G^{\bullet}\right)$ and with all the differentials equal to 0 . 
6.3. Example (Eilenberg-Maclane bundles). Let us consider the particular case when $G^{\bullet}$ consists of only one linear strand. In order to do that, it is convenient to recall another functor considered in [2]. Namely, let $S-$ mod be the category of finitely generated graded $S$-modules. One defines a functor $\mathrm{G}: S-\bmod \rightarrow$ $\mathrm{C}^{+}(\bmod -\Lambda)$ by associating to each graded $S$-module $M$ a complex $\mathrm{G}(M)$ whose $p$ th term is $\mathrm{G}(M)^{p}:=M_{p} \otimes \Lambda^{\vee}(p)$ and whose $p$ th differential $d_{\mathrm{G}(M)}^{p}$ is induced by the multiplication map $M_{p} \otimes V^{*} \rightarrow M_{p+1}$. If $M$ has finite length and $0<j<n$, then $G^{\bullet}:=\mathrm{G}(M)(-j)$ is an HT-complex. In this case, let $\mathcal{E}$ be the vector bundle corresponding to $G^{\bullet}$ as in 6.1, and let $\mathcal{L}^{\bullet}$ be the complex obtained from $\mathrm{L}\left(G^{\bullet}\right)$ as in Remark 6.2. Using Remark 1.8, $\mathrm{L}\left(G^{\bullet}\right)^{m}=0$ for $m>j$ or for $m<j-n-1$, hence the same is true for $\mathcal{L}^{\bullet}$. It follows that, if

$$
0 \rightarrow F^{-n-1} \rightarrow \cdots \rightarrow F^{-1} \rightarrow F^{0} \rightarrow M \rightarrow 0
$$

is a minimal free resolution of $M$ in $S-\bmod$, then $\Gamma_{*} \mathcal{L}^{\bullet} \cong \mathrm{T}^{-j} F^{\bullet}$ and $\mathcal{E}$ is a sheafification of $\operatorname{Ker}\left(F^{-j} \rightarrow F^{-j+1}\right)$. The vector bundles of this kind are called Eilenberg-Maclane bundles by Horrocks [10].

6.4. Remark. Under some assumptions, the vector bundle $\mathcal{E}$ from the statement of Proposition 6.1 is the cohomology of a Beilinson monad obtained directly from the HT-complex $G^{\bullet}$. More precisely, assume that $H^{p}\left(G^{\bullet}\right)_{-1-p}=0$ for all $p<0$, and $H^{p}\left(G^{\bullet}\right)_{-p}=0$ for all $p>0$. Let $C^{\bullet}$ be the complex defined by $C^{p}:=\mathcal{Z}^{0} \mathrm{~L}\left(G^{p}\right) \cong$ $\bigoplus_{p-i} H_{p-i}^{i} \otimes \Omega^{i-p}(i-p)$ and with the differential $d_{C}^{p}:=\mathcal{Z}^{0} \mathrm{~L}\left(d_{G}^{p}\right)$ for all $p \in \mathbb{Z}$. $0<i<n$

Then $\mathcal{H}^{0}\left(C^{\bullet}\right) \cong \mathcal{E}$ and $\mathcal{H}^{i}\left(C^{\bullet}\right)=0$ for $i \neq 0$.

Indeed, we shall use the method of Eisenbud et al. 4], (6.1) for deriving Beilinson monads from Tate resolutions. Let $I^{\bullet}$ be a Tate resolution of $\mathcal{E}$ and consider the complex $\tilde{C}^{\bullet}$ defined by $\tilde{C}^{p}:=\mathcal{Z}^{0} \mathrm{~L}\left(I^{p}\right) \cong \bigoplus_{0 \leq i \leq n} \mathrm{H}^{i} \mathcal{E}(p-i) \otimes \Omega^{i-p}(i-p)$ and with the differential $d_{\tilde{C}}^{p}:=\mathcal{Z}^{0} \mathrm{~L}\left(d_{I}^{p}\right)$ for all $p \in \mathbb{Z}$. Then, by [4], (6.1) (see, also, the proof of [3], (12)), $\mathcal{H}^{0}\left(\tilde{C}^{\bullet}\right) \cong \mathcal{E}$ and $\mathcal{H}^{i}\left(\tilde{C}^{\bullet}\right)=0$ for $i \neq 0$. Now, consider the morphisms of complexes $G^{\bullet} \rightarrow I^{\bullet} / F_{0} I^{\bullet} \leftarrow I^{\bullet}$. Using the notations from Remark 6.2. our additional assumptions imply that

$$
\mathcal{L}^{-1} \cong \bigoplus_{p \geq 0} H^{p}\left(G^{\bullet}\right)_{-1-p} \otimes \mathcal{O}(-1-p) \quad \text { and } \quad \mathcal{L}^{0} \cong \bigoplus_{p \leq 0} H^{p}\left(G^{\bullet}\right)_{-p} \otimes \mathcal{O}(-p)
$$

hence $\mathrm{H}^{0} \mathcal{E}=0$ and $\mathrm{H}^{0} \mathcal{E}^{*}(-1)=0$. It follows that $\mathrm{H}^{0} \mathcal{E}(i)=0$ for all $i \leq 0$ and, by Serre duality, $\mathrm{H}^{n} \mathcal{E}(i)=0$ for all $i \geq-n$. But this implies that one gets isomorphisms $C^{p}=\mathcal{Z}^{0} \mathrm{~L}\left(G^{p}\right) \stackrel{\approx}{\longrightarrow} \mathcal{Z}^{0} \mathrm{~L}\left(I^{p} / F_{0} I^{p}\right) \stackrel{\approx}{\approx} \mathcal{Z}^{0} \mathrm{~L}\left(I^{p}\right)=\tilde{C}^{p}$, for all $p \in \mathbb{Z}$.

6.5. Remark. It follows from the main results of this paper that the vector bundle $\mathcal{E}$ from the statement of Proposition 6.1 is indecomposable if and only if the corresponding HT-complex $G^{\bullet}$ is indecomposable. This has an amusing consequence: let $\mathcal{E}$ be an indecomposable vector bundle on $\mathbb{P}_{n}$ and let $m \in \mathbb{Z}$. If $\mathrm{H}^{i} \mathcal{E}(m-i)=0$ for $0<i<n$, then $\mathrm{H}^{i} \mathcal{E}\left(m^{\prime}-i\right)=0$ for $0<i<n$ and for either every $m^{\prime} \geq m$ or every $m^{\prime} \leq m$.

Indeed, let $G^{\bullet}$ be the HT-complex corresponding to the stable isomorphism class of $\mathcal{E}$. Our assumption implies that $G^{m}=0$, hence $G^{\bullet}=\sigma^{>m} G^{\bullet} \oplus \sigma^{<m} G^{\bullet}$. One deduces that either $\sigma^{>m} G^{\bullet}=0$ or $\sigma^{<m} G^{\bullet}=0$. 
6.6. Remark. It seems impractical to use the results from this section to construct vector bundles. However, one can, at least, recuperate some of the well-known vector bundles of small rank on projective spaces. The following method is essentially due to Horrocks. Consider an HT-complex $G^{\bullet}$ with only two non-zero terms, $G^{0}$ and $G^{-1}$. It has only one non-zero differential, $d^{-1}: G^{-1} \rightarrow G^{0}$. Let $\mathcal{E}$ be the vector bundle associated to $G^{\bullet}$ as in 6.1. Assume that the conditions from Remark 6.4 are satisfied, i.e., that $d_{0}^{-1}: G_{0}^{-1} \rightarrow G_{0}^{0}$ is injective. In this case, if $\mathcal{L}^{\bullet}$ is the complex from Remark 6.2, then

$$
\mathcal{L}^{-1} \cong H^{0}\left(G^{\bullet}\right)_{-1} \otimes \mathcal{O}(-1) \quad \text { and } \quad \mathcal{L}^{0} \cong H^{0}\left(G^{\bullet}\right)_{0} \otimes \mathcal{O} \oplus H^{-1}\left(G^{\bullet}\right)_{1} \otimes \mathcal{O}(1)
$$

and the linear part of the differential $\mathcal{L}^{-1} \rightarrow \mathcal{L}^{0}$ is defined by the multiplication $\operatorname{map} H^{0}\left(G^{\bullet}\right)_{-1} \otimes V \rightarrow H^{0}\left(G^{\bullet}\right)_{0}$.

Let $H \subset H^{0}\left(G^{\bullet}\right)_{-1}$ (resp., $\left.H^{0}\left(G^{\bullet}\right)_{0} \rightarrow K\right)$ be a sub- (resp., quotient) vector space, and consider the induced morphisms $\mu: H \otimes \mathcal{O}(-1) \rightarrow \mathcal{E}$ and $\varepsilon: \mathcal{E} \rightarrow K \otimes \mathcal{O}$. Then $\varepsilon \circ \mu=0$ if and only if the composite map $H \otimes V \rightarrow H^{0}\left(G^{\bullet}\right)_{0} \rightarrow K$ is 0 . Moreover, Lemma 6.7 below gives conditions under which $\mu$ is a monomorphism of vector bundles and $\varepsilon$ is an epimorphism. If these conditions are satisfied, then we get a monad

$$
0 \longrightarrow H \otimes \mathcal{O}(-1) \longrightarrow \mathcal{E} \longrightarrow K \otimes \mathcal{O} \longrightarrow 0
$$

whose cohomology is a vector bundle of $\operatorname{rank} \operatorname{rank}(\mathcal{E})-\operatorname{dim}(H)-\operatorname{dim}(K)$.

6.7. Lemma. Under the assumptions of Remark 6.6

(a) $\mu$ is a monomorphism of vector bundles if and only if $H$ intersects the image of the bilinear multiplication map $H^{0}\left(G^{\bullet}\right)_{-2} \times V \rightarrow H^{0}\left(G^{\bullet}\right)_{-1}$ only in 0 .

(b) $\varepsilon$ is an epimorphism if and only if the composite map $H^{0}\left(G^{\bullet}\right)_{-1} \otimes V \rightarrow$ $H^{0}\left(G^{\bullet}\right)_{0} \rightarrow K$ induces, for every $0 \neq v \in V$, a surjection $H^{0}\left(G^{\bullet}\right)_{-1} \otimes v \rightarrow$ $K$.

(c) Assume, furthermore, that $d_{0}^{-1}$ is an isomorphism, hence $H^{0}\left(G^{\bullet}\right)_{0}=0$. Consider a quotient vector space $H^{-1}\left(G^{\bullet}\right)_{1} \rightarrow Q$ and the induced morphism $\pi: \mathcal{E} \rightarrow Q \otimes \mathcal{O}(1)$. Then $\pi$ is an epimorphism if and only if the subspace $Q^{*} \subset\left(H^{-1}\left(G^{\bullet}\right)_{1}\right)^{*}$ intersects the image of the bilinear multiplication map $\left(H^{-1}\left(G^{\bullet}\right)_{2}\right)^{*} \times V \rightarrow\left(H^{-1}\left(G^{\bullet}\right)_{1}\right)^{*}$ only in 0 . We are unable to state, in this case, a condition equivalent to $\pi \circ \mu=0$.

Proof. For (a) one uses the exact sequence

$$
H^{0}\left(G^{\bullet}\right)_{-2} \otimes \mathcal{O}(-2) \longrightarrow H^{0}\left(G^{\bullet}\right)_{-1} \otimes \mathcal{O}(-1) \longrightarrow \mathcal{E} \longrightarrow 0,
$$

for (b) the fact that $H^{0}\left(G^{\bullet}\right)_{-1} \otimes \mathcal{O}(-1) \rightarrow \mathcal{E}$ is an epimorphism, and for (c) the exact sequence

$$
0 \longrightarrow \mathcal{E} \longrightarrow H^{-1}\left(G^{\bullet}\right)_{1} \otimes \mathcal{O}(1) \longrightarrow H^{-1}\left(G^{\bullet}\right)_{2} \otimes \mathcal{O}(2) .
$$

6.8. Example (Trautmann [14, Vetter [16], Tango [13]). Take $G^{\bullet}=\Lambda^{\vee}(-j)$ for some $0<j<n$, hence $\mathcal{E}=\Omega_{\mathbb{P}}^{j}(j)$. Consider a vector subspace $H \subseteq G_{-1}^{0}=\Lambda^{j+1} V^{*}$. Lemma 6.7. (a) tells us, in this case, that the evaluation morphism $H \otimes \mathcal{O}(-1) \rightarrow$ $\Omega_{\mathbb{P}}^{j}(j)$ is a monomorphism of vector bundles if and only if the $H$ intersects the image of the bilinear contraction map $\Lambda^{j+2} V^{*} \times V \rightarrow \Lambda^{j+1} V^{*}$ only in 0 . It is convenient to use the identifications $\Lambda^{j+1} V^{*} \cong \Lambda^{n-j} V$ and $\Lambda^{j+2} V^{*} \cong \Lambda^{n-j-1} V$. 
Then the above contraction map can be identified (up to sign) with the exterior multiplication $\Lambda^{n-j-1} V \times V \rightarrow \Lambda^{n-j} V$.

Assume, now, that $j=n-2$. The image of the bilinear map $V \times V \rightarrow \Lambda^{2} V$ is the affine cone over the Plücker embedding of $\operatorname{Grass}_{2}(V)$, hence its dimension is $2 n-1$. It follows that the largest possible dimension of $H$ for which $H \otimes \mathcal{O}(-1) \rightarrow$ $\Omega_{\mathbb{P}}^{n-2}(n-2)$ is a monomorphism of vector bundles is $\left(\begin{array}{c}n+1 \\ 2\end{array}\right)-(2 n-1)=\left(\begin{array}{c}n-1 \\ 2\end{array}\right)$. In this case, the rank of the cokernel $\mathcal{F}$ is $n-1$.

One can construct concrete examples of such subspaces $H$ of $\Lambda^{2} V$. For instance, if $e_{0}, \ldots, e_{n}$ form a basis of $V$, one may take for $H$ the subspace spanned by the elements

$$
w_{i j}= \begin{cases}e_{i} \wedge e_{j}-e_{0} \wedge e_{i+j} & \text { for } i+j \leq n, \\ e_{i} \wedge e_{j}-e_{i+j-n} \wedge e_{n} & \text { for } i+j>n,\end{cases}
$$

where $0<i<j<n$. Since $\Omega_{\mathbb{P}}^{n-2}(n-2)$ has a resolution

$0 \rightarrow \Lambda^{n+1} V^{*} \otimes \mathcal{O}(-3) \rightarrow \Lambda^{n} V^{*} \otimes \mathcal{O}(-2) \rightarrow \Lambda^{n-1} V^{*} \otimes \mathcal{O}(-1) \rightarrow \Omega^{n-2}(n-2) \rightarrow 0$

or, equivalently, a resolution

$$
0 \rightarrow \mathcal{O}(-3) \rightarrow V \otimes \mathcal{O}(-2) \rightarrow \Lambda^{2} V \otimes \mathcal{O}(-1) \rightarrow \Omega^{n-2}(n-2) \rightarrow 0,
$$

it follows that $\mathcal{F}$ has a resolution

$$
0 \rightarrow \mathcal{O}(-3) \rightarrow V \otimes \mathcal{O}(-2) \rightarrow\left(\Lambda^{2} V / H\right) \otimes \mathcal{O}(-1) \rightarrow \mathcal{F} \rightarrow 0 .
$$

Now, let $X_{0}, \ldots, X_{n}$ be the dual basis of $V^{*}$. Choosing a convenient (and rather obvious) basis for the subspace $\left(\Lambda^{2} V / H\right)^{*}$ of $\Lambda^{2} V^{*}$ (for the particular $H$ considered above), one easily sees that $\mathcal{F}^{*}$ is the vector bundle constructed by $\mathrm{U}$. Vetter [16] using an explicit matrix of linear forms.

6.9. Example (Horrocks [10]). Assume that $G^{0}=\Lambda^{\vee}(-i)$ and $G^{-1}=\Lambda^{\vee}(-j-1)$, for some $0<i<j<n$. The differential $d^{-1}: G^{-1} \rightarrow G^{0}$ is defined by a linear function $\Lambda^{j-i+1} V^{*} \rightarrow k$, i.e., by an element $\omega \in \Lambda^{j-i+1} V$. Let $-\cdot-: \Lambda^{\vee} \times \Lambda \rightarrow \Lambda^{\vee}$ denote the bilinear map defining the structure of right $\Lambda$-module on $\Lambda^{\vee}$, i.e., the contraction map. Then $d_{0}^{-1}: G_{0}^{-1} \rightarrow G_{0}^{0}$ is just $-\cdot \omega: \Lambda^{j+1} V^{*} \rightarrow \Lambda^{i} V^{*}$, which can be identified (up to sign) with $-\wedge \omega: \Lambda^{n-j} V \rightarrow \Lambda^{n-i+1} V$.

Let us give, following Horrocks [10], some examples of elements $\omega$ for which $d_{0}^{-1}$ is injective. Let $e_{0}, \ldots, e_{n}$ be a $k$-basis of $V$ and let $X_{0}, \ldots, X_{n}$ be the dual basis of $V^{*}$. Assume $n$ is odd, $n=2 m-1$, and consider the elements

$$
\alpha=\sum_{i=0}^{m-1} e_{i} \wedge e_{m+i} \in \Lambda^{2} V \quad \text { and } \quad \beta=\sum_{i=0}^{m-1} X_{i} \wedge X_{m+i} \in \Lambda^{2} V^{*} .
$$

Let $\alpha^{(i)}=\frac{1}{i !} \alpha^{\wedge i} \in \Lambda^{2 i} V$ be the $i$ th divided power of $\alpha$, for $0<i<m$. Then $-\cdot \alpha^{(m-i)}: \Lambda^{2 m-i} V^{*} \rightarrow \Lambda^{i} V^{*}$ can be identified with $\beta^{(i)} \cdot-: \Lambda^{i} V \rightarrow \Lambda^{i} V^{*}$ via $\Lambda^{2 m-i} V^{*} \cong \Lambda^{i} V$. For $i=1, \varphi:=\beta \cdot-: V \rightarrow V^{*}$ is an isomorphism. But, for $i>1$, it is not so easy to show that $\beta^{(i)} \cdot-$ is an isomorphism (in fact, one needs some assumptions on chark). The point is that $\beta^{(i)} \cdot-$ is not equal to $\Lambda^{i} \varphi$ times a non-zero constant. Assume, for simplicity, that chark $=0$. Consider the endomorphisms $A=-\cdot \alpha$ and $B=\beta \wedge-$ of the $k$-vector space $\Lambda^{\vee}$ and let $C=[A, B]$. Then one can check that, for $\eta \in\left(\Lambda^{\vee}\right)_{-p}=\Lambda^{p} V^{*}, C \eta=(m-p) \eta$. It follows that $[C, A]=2 A$ and $[C, B]=-2 B$, hence $A, B, C$ define a representation of Lie algebras $\rho: s l_{2} \rightarrow g l\left(\Lambda^{\vee}\right.$ ) (see, for example, [8], pp. 118-120). One deduces that $A^{i}: \Lambda^{m+i} V^{*} \rightarrow \Lambda^{m-i} V^{*}$ is an isomorphism for $0 \leq i \leq m$. 
We shall denote, for later use, by $\mathcal{E}_{i}, 0<i<m$, the bundle obtained from the above $G^{\bullet}$ as in 6.1 for $n=2 m-1, j=2 m-i-1$ and $\omega=\alpha^{(m-i)}$. The bundle $\mathcal{E}_{1}$ is the so-called null-correlation bundle and has rank $n-1$ on $\mathbb{P}_{n}, n$ odd.

6.10. Example (Famous vector bundles). The Horrocks-Mumford rank 2 vector bundle on $\mathbb{P}_{4}$, [12, can be obtained by the method from Remark 6.6 and Lemma 6.7 starting with $G^{\bullet}=k^{2} \otimes \Lambda^{\vee}(-2)$. The Sasakura rank 3 vector bundle on $\mathbb{P}_{4}$, [1] can also be obtained in this way starting with a certain HT-complex $G^{\bullet}$ with $G^{0}=\Lambda^{\vee}(-1) \oplus \Lambda^{\vee}(-2)$ and $G^{-1}=\Lambda^{\vee}(-3)$.

More complicated, from the point of view of these general considerations, is the construction of the Horrocks rank 3 vector bundle on $\mathbb{P}_{5}$, [11. Assume $n=5$, and consider the vector bundle $\mathcal{E}_{2}$ defined at the end of Example 6.9 (which exists, in fact, if chark $\neq 2$ ). Since $d_{0}^{-1}: G_{0}^{-1} \rightarrow G_{0}^{0}$ is an isomorphism, the morphism $\delta^{\prime}: \mathcal{G}^{-1} \rightarrow \mathcal{G}^{0}$ from the proof of Proposition 6.1 is the composite morphism

$$
G_{-1}^{0} \otimes \mathcal{O}(-1) \longrightarrow G_{0}^{0} \otimes \mathcal{O} \stackrel{\approx}{\longleftarrow} G_{0}^{-1} \otimes \mathcal{O} \longrightarrow G_{1}^{-1} \otimes \mathcal{O}(1) .
$$

One has $G_{-1}^{0}=\Lambda^{3} V^{*}=G_{1}^{-1}$, a decomposition $\Lambda^{3} V^{*}=B_{-1}^{0} \oplus Z_{1}^{-1}$ and $P:=Z_{1}^{-1}$ is the set of primitive elements $\eta$ of $\Lambda^{3} V^{*}$, i.e., those with $A \eta=0$. If $\eta \in P$ and $v \in V$, then $\eta \cdot v$ is a primitive element of $\Lambda^{2} V^{*}$ hence $A B(\eta \cdot v)=\eta \cdot v$. This means that $d_{0}^{-1}(\beta \wedge(\eta \cdot v))=\eta \cdot v$. It follows that the differential $\mathcal{L}^{-1} \rightarrow \mathcal{L}^{0}$ of the complex $\mathcal{L}^{\bullet}$ from Remark 6.2 can be identified with the morphism $P \otimes \mathcal{O}(-1) \rightarrow P \otimes \mathcal{O}(1)$ whose reduced fibre at $[v] \in \mathbb{P}_{5}$ is $P \rightarrow P, \eta \mapsto(\beta \cdot v) \wedge(\eta \cdot v)$.

Now let $\mu: \mathcal{O}(-1) \rightarrow \mathcal{E}_{2}$ be the morphism defined by the element $X_{0} \wedge X_{1} \wedge$ $X_{2}+X_{3} \wedge X_{4} \wedge X_{5} \in P$ and let $\pi: \mathcal{E}_{2} \rightarrow \mathcal{O}(1)$ be the morphism defined by the linear function $P \rightarrow k$ induced by $e_{0} \wedge e_{1} \wedge e_{2}+e_{3} \wedge e_{4} \wedge e_{5} \in \Lambda^{3} V$. Using the above description of the differential $\mathcal{L}^{-1} \rightarrow \mathcal{L}^{0}$, one checks that $\pi \circ \mu=0$. Moreover, one can check, using Lemma 6.7. (a),(c), that $\mu$ is a monomorphism of vector bundles and $\pi$ an epimorphism. Consequently, $\mu$ and $\pi$ define a monad

$$
0 \longrightarrow \mathcal{O}(-1) \longrightarrow \mathcal{E}_{2} \longrightarrow \mathcal{O}(1) \longrightarrow 0
$$

whose cohomology is a rank 3 vector bundle on $\mathbb{P}_{5}$.

\section{REFERENCES}

[1] H. Abo, W. Decker, N. Sasakura, An elliptic conic bundle in $\mathbb{P}^{4}$ arising from a stable rank-3 vector bundle, Math. Z. 229 (1998), 725-741. MR1664785(2001b:14071)

[2] I.N. Bernstein, I.M. Gel'fand, S.I. Gel'fand, Algebraic bundles over $\mathbb{P}^{n}$ and problems of linear algebra, Funktsional'nyi Analyz i Ego Prilozheniya 12, No. 3 (1978), 66-67. MR0509387 (80c:14010a)

[3] I. Coandă, On the Bernstein-Gel'fand-Gel'fand correspondence and a result of Eisenbud, Fløystad, and Schreyer, Journ. Math. Kyoto Univ. 43 (2003), 429-439. MR2051032

[4] D. Eisenbud, G. Fløystad, F.-O. Schreyer, Sheaf cohomology and free resolutions over exterior algebras, Transactions of the AMS 355 (2003), 4397-4426. MR:1990756 (2004f:14031)

[5] G. Fløystad, Describing coherent sheaves on projective spaces via Koszul duality, preprint math.AG/0012263.

[6] G. Fløystad, Koszul duality and equivalences of categories, preprint math.RA/0012264.

[7] S.I. Gel'fand, Sheaves on $\mathbb{P}^{n}$ and problems of linear algebra, appendix to the Russian edition of the book Ch. Okonek, M. Schneider, H. Spindler: Vector Bundles on Complex Projective Spaces, Birkhäuser 1980, Mir, Moscow 1984. MR0561910|(81b:14001) MR0778380 (86i:14005)

[8] Ph. Griffiths, J. Harris, Principles of algebraic geometry, John Wiley \& Sons, Inc., New York 1978. MR0507725 (80b:14001) 
[9] G. Horrocks, Vector bundles on the punctured spectrum of a local ring, Proc. London Math. Soc. 14 (1964), 689-713. MR0169877 (30:120)

[10] G. Horrocks, Construction of bundles on $\mathbb{P}^{n}$, In: A. Douady and J.-L. Verdier (eds.) Les équations de Yang-Mills, Séminaire E.N.S. (1977-1978), Astérisque 71-72, Soc. Math. de France (1980), 197-203. MR0589891 (84j:14026)

[11] G. Horrocks, Examples of rank three vector bundles on five-dimensional projective space, J. London Math. Soc. 18 (1978), 15-27. MR0502651 (80d:14011)

[12] G. Horrocks, D. Mumford, A rank 2 vector bundle on $\mathbb{P}^{4}$ with 15,000 symmetries, Topology 12 (1973), 63-81. MR0382279 (52:3164)

[13] H. Tango, An example of indecomposable vector bundle of rank $n-1$ on $\mathbb{P}^{n}$, J. Math. Kyoto Univ. 16, No. 1 (1976), 201-207. MR0401766 (53:5593)

[14] G. Trautmann, Darstellung von Vektorraumbündeln über $\mathbb{C}^{n} \backslash\{0\}$, Archiv der Mathematik (Basel) 24 (1973), 303-313. MR0352523 (50:5010)

[15] G. Trautmann, Moduli of Vector Bundles on $\mathbb{P}_{n}(\mathbb{C})$, Math. Ann. 237 (1978), 167-186. MR0507912 (80a:14008)

[16] U. Vetter, Zu einem Satz von G. Trautmann über den Rang gewisser kohärenter analytischer Moduln, Archiv der Mathematik (Basel) 24 (1973), 158-161. MR0344518 (49:9257)

Institute of Mathematics of the Romanian ACAdemy, P.O. Box 1-764, RO-70700 Bucharest, Romania

E-mail address: Iustin.Coanda@imar.ro

Fachbereich Mathematik, Universität Kaiserslautern, Erwin-Schrödinger-Strasse, D-67663 Kaiserslautern, Germany

E-mail address: trm@mathematik.uni-kl.de 